\title{
El liderazgo y su relación con la satisfacción de los clientes: un enfoque teórico
}

\section{Leadership and its relationship with customer satisfaction: a theoretical approach}

Stefanie Desiré Villanueva Sampín

Osvaldo Santiago Carrera Soria

Escuela Superior Politécnica del Litoral, Ecuador

María Auxiliadora Guerrero Bejarano, MBA

Universidad Internacional del Ecuador, Ecuador

Autor para correspondencia: svillanu@espol.edu.ec; scingcarrera@gmail.com; maguerrerobe@uide.edu.ec

Fecha de recepción: 30 de agosto de 2018 - Fecha de aceptación: 01 de noviembre de 2018

\section{Resumen}

En toda organización es importante que cada grupo tenga una estructura establecida, en donde siempre existirán quienes asuman un mayor grado de responsabilidad, evalúen, tomen decisiones y tengan su enfoque basado en los objetivos organizacionales. Por ello, es muy natural que en cualquier grupo de trabajo sobresalga un líder, el cual contribuye con el desarrollo y logro de los objetivos establecidos.

Existen varios tipos de liderazgo, pero sea cual sea el perfil del mismo, el objetivo principal deberá ir encaminado a dirigir al grupo hacia el éxito y en el entorno empresarial no es una excepción. Para que el cliente perciba un buen servicio y se encuentre satisfecho con el mismo, el empleado deberá sentirse motivado, es por ello la importancia de la figura del líder.

Palabras claves: liderazgo; decisiones; motivación; satisfacción de cliente

\begin{abstract}
In every organization it is important that each group has an established structure, where there will always be those who assume a greater degree of responsibility, evaluate, make decisions and have their approach based on organizational objectives. Therefore, it is very natural that in any work group a leader stands out, which contributes to the development and achievement of the established objectives.

There are several types of leadership, but whatever the profile of the same, the main objective should be aimed at leading the group towards success and in the business, environment is not an exception. For the client to perceive a good service and be sati sfied with it, the employee must feel motivated, that is why the importance of the figure of the leader.
\end{abstract}

Key words: leadership; decisions; motivation; customer satisfaction 


\section{Introducción}

Actualmente, en las organizaciones se utilizan diversos métodos para gestionar todo tipo de aspectos o enfoques necesarios en el entorno empresarial. Tales como la implementación de sistemas de gestión en donde se requiere de líderes que orienten a la organización a la consecución de los objetivos establecidos, es por ello, que el liderazgo es de suma importancia en cualquier tipo de empresa. Cuando existe un liderazgo eficaz dentro de la organización, las personas se sienten motivadas y comprometidas en relación a las metas de la empresa, disminuyendo la comunicación deficiente que suelen originarse en los distintos niveles jerárquicos.

Es de suma importancia generar una gestión de calidad adecuada de forma que nuestros clientes externos no sean los únicos beneficiados de esto sino también el cliente interno, los cuales tienen un alto grado de significancia ya que se encuentran estrechamente relacionados con la calidad del servicio que brinda la organización al cliente externo. Por ello, los directivos tienen un papel decisivo para que las organizaciones sean capaces de motivar a las personas necesarias ya que el tener un personal motivado por el trabajo que desempeña brindará un servicio de calidad al cliente satisfaciendo sus necesidades. En general en las empresas, se producen insatisfacciones de su personal lo que influye de manera negativa disminuyendo su desempeño individual y organizacional afectando directamente en la calidad del servicio, muchas empresas fracasan por la falta de un buen líder y no dar la importancia necesaria a los beneficios que nos brinda entregar servicios de calidad a los clientes.

\section{Estilos de Liderazgo, Satisfacción del Cliente}

En la variedad de ámbitos de la vida de todo ser humano sea académico, social o laboral ha surgido la necesidad de afrontar los desafíos constantes que el mundo impone tanto a las organizaciones como a los individuos, por tanto, es tarea de un líder lograr metas y enfrentar dichos desafíos junto a su personal a cargo, haciendo referencia a Mazón (2012). Dichos estilos se verán reflejados directamente en el desempeño de los colaboradores de una organización y a través de dichos colaboradores se llega a los clientes y su satisfacción como resultado final (Acuña \& Huamani, 2010) que describen dicha satisfacción en función de la respuesta emocional del cliente al comparar el servicio recibido contra el esperado, y expresan que un cliente satisfecho contribuye a la rentabilidad de la organización.

Considerando de a partir de un liderazgo optimo se logra colaboradores satisfechos, colaboradores más productivos y que ofrecen un mejor servicio al cliente (Chuang, Judge \& Liaw, 2012), se aprecia la relación entre el estilo de liderazgo y la satisfacción del cliente. En este contexto, han surgido un sin número de estilos de liderazgo, algunos como el autoritario donde el líder toma las decisiones por su cuenta o liderazgo autócrata que se basa solo en la figura del líder como un todo, que solo busca la obediencia y adhesión a sus decisiones (Mazón, 2012).

El liderazgo democrático o participativo, como explica Acuña (2010), es otro estilo y es aquel que cuenta con las opiniones del resto de integrantes para determinar la decisión final, fomentando la satisfacción en tareas que involucran el ego de los colaboradores. 
Muy similar, pero sin ser lo mismo, el liderazgo liberal, donde el líder con una conducta pasiva, delega su liderazgo en los demás (Mazón, 2012) y donde los colaboradores asumen la responsabilidad por su propia motivación guía y control, con características similares al liderazgo orientado por resultados, donde el líder busca objetivos y retos, mostrando confianza en su grupo de colaboradores (Acuña, 2010).

El liderazgo Solidario, se concentra en las necesidades de colaboradores y promueve un clima laboral adecuado a criterio de Acuña (2010), considerándolo el más adecuado para situaciones estresantes o frustrantes. Podemos acotar que dándoles emociones agradables a los colaboradores se producen resultados positivos como una retroalimentación facial con el cliente como indica Chuang (2012). Otro estilo de liderazgo es el transaccional, donde el líder se limita a aportar los recursos que considera válidos para el grupo y el directivo donde el líder explica lo que se debe hacer como hace referencia Acuña (2010).

El liderazgo transformacional, poco investigado como indican Chuang, Judge \& Liaw (2012), quien inspira y motiva a los seguidores para hacer sacar lo mejor de ellos mismos. En donde, para muchos autores este último estilo de liderazgo es considerado como el más efectivo, dada la sinergia de emociones positivas o agradables con la satisfacción laboral del empelado.

No todos los líderes se encuentran preparados para aplicar este estilo de liderazgo, un líder transformacional ha de ser inteligente, apasionado, con una visión y misión clara y capaz de transmitirla e interiorizarla en todos los niveles de la organización (Chuang, 2012). Según diversos estudios, los equipos dirigidos con este estilo, son aquellos que obtienen mejores resultados, los que presentan una mayor motivación y permiten que exista un mejor clima laboral.

La relación entre los estilos de liderazgo y la satisfacción del cliente está mediada en parte por el rendimiento de los empleados considerando sus estados de ánimo y emociones como establece Chuang (2012) y esa es la razón principal por la que el liderazgo bajo los que ellos se encuentren juega un rol importante en su desempeño frente al cliente externo, ya que es un réplica de la cultura organizacional dentro de sus equipos de trabajo. Es decir, un impacto positivo mostrado desde alta dirección será desplegado hacia todos los niveles de la organización, mostrando un alto nivel de iniciación y compromiso para con sus clientes internos que son cada uno de los colaboradores, lo que los llevará a comprender el valor de la orientación al cliente y apropiarse de ello.

Los líderes son una parte fundamental para motivar al personal y generar en ellos emociones positivas (Chuang, 2012) como también para conseguir que el equipo salga adelante. Es imprescindible un buen liderazgo ya que esto podría ocasionar que se pierda el rumbo hacia el éxito y muy difícilmente adquieran compromiso con los colaboradores peor aún que estos desconozcan los objetivos que se buscan (Mazón, 2012). Es por esta razón, que un líder tiene que convertirse en un referente para que de esta manera tenga un impacto positivo en el servicio otorgado al cliente y este pueda obtener un servicio de calidad y por ende se obtenga la satisfacción del mismo. 
La calidad de un servicio o producto tiende a ser subjetiva ya que es exclusiva de los parámetros de evaluación o percepción que pueda tener cada persona; sin embargo, siempre se debe tener controlada la satisfacción del mismo, ya que es el veredicto del servicio recibido ante la expectativa mantenida versus el desempeño real del servicio/producto de una organización. Por ello, la satisfacción de los consumidores determinará sus relaciones futuras con la organización, es decir, si usuario experimenta satisfacción alta, definitivamente incrementará su fidelidad con la organización, sin importar otras ofertas, recomendará el servicio y será la mejor publicidad del mismo.

La satisfacción del cliente posterior a la compra de un producto o la prestación de un servicio, está relacionado directamente a su conclusión sobre la oferta en función de sus expectativas previas. Lo que significa que, si sus expectativas superan lo percibido, el cliente queda insatisfecho; por otra parte, si sus conclusiones resultantes están a la altura de sus expectativas, el cliente queda satisfecho, pero si los resultados superan sus expectativas, el cliente queda muy satisfecho o encantado y es ahí cuando nuestro servicio empieza a tener una ventaja competitiva. Lo que nos lleva a que, si la organización desea satisfacer a sus clientes, es imprescindible que posea la capacidad de alinear sus servicios con lo que esperan o desean sus clientes de ellos, lo que puede significar la experiencia otorgada. A mayor alineación, habrá una mejor percepción de satisfacción dentro y fuera de la organización.

Sin embargo, aun cuando es primordial que se dirijan los esfuerzos en la satisfacción de los clientes, es importante resaltar que las organizaciones deben velar por satisfacer a sus partes interesadas, quienes se ven afectadas por las decisiones tomadas y a su vez también pueden afectar a las misma, tales como lo son los accionistas, colaboradores, alianzas, proveedores y comunidad; ya que de todos ellos y en diferente magnitud radica el éxito de la empresa cuyos resultados organizacionales son una consecuencia del nivel de servicio y satisfacción de los clientes, que se obtiene mediante el liderazgo de la calidad total apoyado en los pilares de la gestión de personal, de recursos y del sistema de calidad bajo una planificación enfocada al objetivo estratégico de la organización.

\section{Conclusiones}

El resultado directo de un buen liderazgo está estrechamente relacionado con el éxito de la innovación y buen servicio al cliente, mientras que de manera indirecta están asociados a las ventajas que logre la compañía gradualmente, así como el éxito en cuanto al tema económico se refiere reflejado en el desempeño general que llegare a demostrar la organización. El hecho que en la entidad organizacional se incentiven buenas prácticas que sean de vital ayuda con la comunicación, compromiso, motivación y aprendizaje continuo, permite una mejora de los procesos y su gestión demostrando de esta manera que el liderazgo sea cual fuera el estilo impulsa una cultura de calidad relacionada con el servicio al cliente dando soporte a la estructura general de la compañía.

En general, se puede expresar que las empresas que mantengan un buen liderazgo mantienen una fuerte ventaja frente aquellas empresas que no lo posean, es por ello, que la brecha para lograr competitividad y diferenciación en el mercado se reduce al lograr un mejoramiento continuo en cuanto a calidad y servicio al cliente se refiere, donde indica que la 
mala actitud del personal genera inseguridad y poca credibilidad en los clientes afectando rentabilidad y volumen de ventas.

Un líder ha de proyectar confianza en el grupo y debe estar totalmente comprometido con los objetivos organizacionales. El líder debe saber cómo mantener al equipo motivado, exigirles responsabilidades y tener una actitud positiva que les ayude a desempeñarse mejor en cada una de sus tareas, ya que si los empleados de la compañía se encuentran comprometidos y motivados brindarán un mejor servicio al cliente generando una total satisfacción y permitiendo que exista una forma eficaz de diferenciarse al resto de las compañías aportando una mejor estrategia y posicionamiento en el mercado en torno a la calidad, eficiencia y servicio al cliente.

La responsabilidad social de las compañías no debe estar enfocada únicamente en generar empleos o ser rentables, sino que deben estar orientadas a capacitar, motivar, proveer en el sentido de un trabajo significativo y a gusto para todos sus empleados, siendo aquí la figura del líder donde adquiere suma importancia. El líder debe comprender la competencia y valores característicos de una organización para llevarla al éxito, sin sobre pasar los derechos de los demás, sino trabajar como lo debe hacer un buen líder para de esta manera lograr cumplir los objetivos organizacionales promoviendo servicios de calidad y que con lleven a la total satisfacción de los clientes.

\section{Bibliografía}

Lars Borgmann, Jens Rowold \& Kai Christian Bormann (2015). Integrating leadership research: a metaanalytical test of Yukl's meta-categories of leadership"

Angelos Pantouvakis \& Christos Patsiouras, (2016). Exploring the role of leadership style on the service quality-customer satisfaction link: Evidence from a B2B environment, International Journal of Quality and Service Sciences, Vol. 8 Issue: 1, pp.88-101.

Salahat MA \& Majid AHBA (2016). Linking leadership styles to customer satisfaction of Palestinian insurance sector: Mediating role of employee's performance. International Journal of Advanced and Applied Sciences, 3(11): 73-82.

Stock, Ruth \& Hoyer, Wayne. (2002). Leadership Style as Driver of Salespeople's' Customer Orientation. Journal of Market-Focused Management, 5. 355-376.

Jyh-Shen Chiou \& Tung-Zong Chang (2009) The Effect of Management Leadership Style on Marketing Orientation, Service Quality, and Financial Results: A Cross-Cultural Study, Journal of Global Marketing, 22:2, 95-107.

Aichia Chuang, Timothy A. Judge \& Yuann Jun Liaw (2012). Transformational leadership and customer service: A moderated mediation model of negative affectivity and emotion regulation. Psychology Press, an imprint of the Taylor \& Francis Group, an Informa business

Ignacio A. Mendoza Martínez, Jesús F. Uribe Prado, Blanca R. García Rivera (2014).

Liderazgo y su Relación con Variables De Resultado: un Modelo Estructural Comparativo entre Liderazgo Transformacional y Transaccional en una Empresa de Entretenimiento en México, Acta de Investigación Psicológica, Volumen 4, Issue 1, pp. 1412-1429 
Ruth Maria StockWayne D. Hoyer (2002). Leadership Style as Driver of Sales peoples Customer Orientation, Journal of Market-Focused Management 5(4):355-376.

Liria E. Perilla-Toro \& Viviola Gómez-Ortiz (2017). Relación del estilo de liderazgo transformacional con la salud y el bienestar del empleado: el rol mediador de la confianza en el líder, Revista de Psicología del Trabajo y de las Organizaciones, Volumen 33, Issue 2, pp. 95-108

Sharma, Laxmi \& Sakshi (2017). Customer Satisfaction with Service Quality: An Empirical study of Banking Sector, Journal of Management Research, and Vol. 9 Issue: 2, pp. 189- 195.

Peñarreta Cuenca, Dunia Soledad. La influencia de los estilos de liderazgo en los niveles de satisfacción laboral de los empleados del GAD Municipal de Loja. Quito, 2014, 115

p. Tesis (Maestría en Desarrollo del Talento Humano). Universidad Andina Simón Bolívar, Sede Ecuador. Área de Gestión.

Villacís Saul (2012). El liderazgo y su incidencia en el Servicio al cliente de la escuela de Conducción para conductores no Profesionales de Pelileo Escudeconp s.a.

Universidad Técnica de Ambato.

Yumeng Yue, Karyn L. Wang \& Markus Groth (2017). Feeling bad and doing good: the effect of customer mistreatment on service Employee's daily display of helping Behaviors. Personnel Psychology.

Zafar Mohsin, Zafar Sana, Asif Aasia, Hunjra Ahmed Imran and Ahmad H. Mushtaq (2012).

Service Quality, Customer Satisfaction and Loyalty: An Empirical Analysis of Banking Sector in Pakistan. Information Management and Business Review, Vol. 4, No. 3, pp. 159-167.

Fernando Jaramillo, Douglas B. Grisaffe, Lawrence B. Chonko \& James A. Roberts (2009) Examining the Impact of Servant Leadership on Sales Force Performance, Journal of Personal Selling \& Sales Management, 29:3, 257-275.

Fernando J. Pons Verdú \& José Ramos (2012). Influencia de los Estilos de Liderazgo y las Prácticas de Gestión de RRHH sobre el Clima Organizacional de Innovación, Revista de Psicología del Trabajo y de las Organizaciones, 28(2):81-98

Esther Ling-Yee Li, Ben Shaw-Ching Liu \& Sherriff T. K. Luk (2017) Customer Participation Behavior in High- Versus Low-Contact Services: The Multiple Roles of Customer Trust, Journal of Global Marketing, 30:5, 322-341, DOI: 10.1080/08911762.2017.1343886

Transformational leadership and group potency in small military units: The mediating role of group identification and cohesion, Revista de Psicología Del Trabajo y de las Organizaciones, Volume 32 , Issue 3 , pp. $145-152$

Diana M. Escandón-Barbosa \& Andrea Hurtado-Ayala (2016). Influencia de los estilos de liderazgo en el desempeño de las empresas exportadoras colombianas. Universidad ICESI.

Ana María Franco Medina, Mayra Lorena Reyes Salazar, Silvia Lorena \& Cuadrado González (2017). Incidencia de los Estilos de Liderazgo en la Satisfacción de los Colaboradores en Empresas de Servicios del Ecuador 